\title{
VHL loss predicts response to Aurora kinase A inhibitor in renal cell carcinoma cells
}

\author{
XIAO-FEI DING ${ }^{1,2}$, JUN ZHOU $^{2,3}$, GUANG CHEN $^{3,4}$ and YING-LIANG WU ${ }^{1}$ \\ ${ }^{1}$ Department of Pharmacology, Shenyang Pharmaceutical University, Shenyang, Liaoning 110016; \\ ${ }^{2}$ Laboratory for Biological Medicine, School of Medicine; ${ }^{3}$ Institute of Tumor; ${ }^{4}$ Department of Pharmacology, \\ School of Pharmaceutical and Chemical Engineering, Taizhou University, Taizhou, Zhejiang 318000, P.R. China
}

Received December 13, 2017; Accepted May 9, 2018

DOI: $10.3892 / \mathrm{mmr} .2018 .9038$

\begin{abstract}
The majority of molecular targets of anticancer agents are limited to a subset of patients, and therefore identification of more specific biomarkers that can be used to improve clinical outcomes is of increasing interest. The present study showed that von Hippel-Lindau tumor suppressor (VHL) tumor-suppressor activity may influence the therapeutic response to Aurora kinase A (AURKA) inhibitors in human renal cell carcinoma (RCC). VHL protein (pVHL) expression was evaluated by immunoblotting in the human RCC cell lines CAKI, ACHN, 786-O, 769-P and A498. The anti-tumor activities of alisertib, an AURKA-specific chemical inhibitor, were detected by Cell Counting Kit-8 assay in vitro and mouse xenograft model in vivo. Additionally, the VHL-mediated anti-tumor activity was assessed in 769-P and CAKI cells via the loss or gain of VHL. The results revealed that VHL-deficient 786-O, 769-P and A498 cells were sensitive to alisertib. By contrast, alisertib-resistant CAKI and ACHN cells expressed the wild type VHL gene. In addition, rescue or knockdown of VHL was observed to increase or decrease alisertib anti-proliferation activity, respectively, in RCC cells. The inverse correlation between the VHL gene expression profile and alisertib sensitivity was further confirmed in human cancer xenografts models. Taken together, these results suggested that VHL loss could potentially serve as a biomarker for predicting the efficacy of AURKA inhibitors.
\end{abstract}

Correspondence to: Dr Ying-Liang Wu, Department of Pharmacology, Shenyang Pharmaceutical University, 103 Wenhua Road, Shenyang, Liaoning 110016, P.R. China

E-mail: yingliang_1016@163.com

Dr Guang Chen, Department of Pharmacology, School of Pharmaceutical and Chemical Engineering, Taizhou University, 1139 Shi-Fu Avenue, Taizhou, Zhejiang 318000, P.R. China

E-mail: gchen@tzc.edu.cn

Key words: aurora kinase A, von Hippel-Lindau tumor suppressor protein, Alisertib, biomarker, renal cell carcinoma

\section{Introduction}

Around 208,500 new cases of kidney cancer are diagnosed annually worldwide (1). It has been predicted that around 63,340 new cases of kidney cancer will occur, and about 14,970 patients will die from kidney cancer in the United States in 2018 (NIH). In China, the kidney cancer incidence has been rising steadily in recent years (MOHC, Ministry of Health of The People's Republic of China). Renal cell carcinoma (RCC), the most common type of kidney cancer in adults, is responsible for approximately $90-95 \%$ of cases $(2,3)$. As $25-30 \%$ of RCC patients exhibit metastatic spread by the time they are diagnosed, drug treatments targeting metastasis are needed during early disease management. In this regard, Aurora kinase A (AURKA) inhibitors have been shown to inhibit the growth and spread of RCC (4-6).

AURKA, a serine-threonine-specific protein kinase, is a member of the Aurora kinase (AURK) family, the members of which serve as key regulators of mitosis; essential for accurate and equal segregation of genomic material from parent to daughter cells (7). Humans have three classes of Aurora kinases, namely AURKA, AURKB and AURKC. AURKA is a 403 amino-acid protein with a calculated molecular weight of $48 \mathrm{kDa}(8)$. This protein has an N-terminal regulatory domain and C-terminal catalytic domain. Two amino acid sequences, the A-box in the regulatory domain and the destruction-box (D-box) in the catalytic domain, are necessary for recognition of AURKA by the APC/C complex, which mediates its degradation at the end of mitosis or in G1 (9). Phosphorylation of a threonine residue within the activation loop in the catalytic domain is critical for Aurora kinase activity (10-12). AURKA is mainly localized at spindle poles and the mitotic spindle during mitosis, where it regulates centrosomes, spindles and kinetochores. Recent studies have revealed that AURKA is frequently overexpressed in several cancer cells, indicating its involvement in tumor initiation and development (5,13-15). Certain ongoing clinical trials and primary studies are assessing the unique therapeutic potential of AURKA-targeted therapy for RCC. However, RCC is relatively resistant to chemotherapy. Therefore, identifying early-stage predictive biomarkers in RCC patients is urgently needed.

In the present study, we showed that RCC cells with loss of the VHL gene were more sensitive to AURKA inhibitor. 
Moreover, we found that elevated pVHL levels improved AURKA degradation, as a novel mechanism for the role of pVHL in regulating AURKA.

\section{Materials and methods}

Cell culture and reagents. CAKI, ACHN, 786-O, 769-P and A498 cells were obtained from the American Type Culture Collection (ATCC, Manassas, VA, USA) and maintained in appropriate medium as suggested by the ATCC. Cells were incubated in a humidified atmosphere of $95 \%$ air plus $5 \% \mathrm{CO}_{2}$ at $37^{\circ} \mathrm{C}$. Alisertib and MG-132 were obtained from Selleck Chemicals, (Houston, TX, USA). Cycloheximide (CHX) was purchased from Solarbio Science and Technology (Beijing, China)

Immunoblotting. After different types of treatment were performed, cells were harvested and resuspended in RIPA lysis buffer (weak). Equivalent amounts of proteins were analyzed by SDS-PAGE. Appropriate antibodies against VHL $(1: 1,000)$, AURKA (1:1,000; Cell Signaling Technology, Inc., Danvers, MA, USA), GAPDH (1:5,000; Santa Cruz Biotechnology, Inc., Dallas, TX, USA) were used. Proteins were visualized with peroxidase-coupled secondary antibody from Sigma-Aldrich; Merck KGaA, (Darmstadt, Germany), using ECL solution for detection.

Animals and anti-tumor activity assay in vivo. BALB/Ca-nu/nu mice aged 4-5 weeks were obtained from Zhejiang Academy of Medical Sciences. The animals were housed in sterile cages under laminar airflow hoods in a specific pathogen-free room with a 12-h light and 12-h dark schedule, and fed autoclaved chow and water ad libitum. Animal experiments were performed according to the institutional ethical guidelines of animal care and were approved by Taizhou University (no. TZYXY2016-302). The largest diameter of tumors developed in all mice examined is $1.745 \mathrm{~cm}$. shRNA-transfected cells were transplanted subcutaneously into the flanks of nude mice. When the tumor volume reached about $100 \mathrm{~mm}^{3}$, the mice were randomly assigned into control and treatment groups, 8 mice each group. Control groups were given vehicle, and treatment groups received alisertib at the indicated doses per os for five days per week for 3 weeks. Tumor volumes were measured twice per week. After treatment, animals were killed by cervical dissociation, and solid tumors were removed and weighed. The inhibition rate was calculated as [(average tumor weight of vehicle control group/average tumor weight of test group)/average tumor weight of normal saline group] X100\%.

Knockdown of VHL by shRNA. The lentivirus vector pLKO.1 was used. The inserted shRNA-targeting $v h l$ sequence was as follows: 5'-CCGGTATCACACTGCCAGTGTATACCTCGAG GTATACACTGGCAGTGTGATATTTTTG-3'. Lentiviral packaging was conducted in 293T cells following standard procedures. The shRNA was transfected into cells using Lipofectamine 2000 (Invitrogen; Thermo Fisher Scientific, Inc., Waltham, MA, USA) according to the instructions of the manufacturer.

Statistical analysis. The data were analyzed using SPSS v13.0 (SPSS, Inc., Chicago, IL, USA). The results were compared using one-way analysis of variance followed by Dunnett's post hoc test for multiple comparisons. All results are expressed as the mean \pm standard deviation from three replicates. $\mathrm{P}<0.05$ was considered to indicate a statistically significant difference.

\section{Results}

VHL gene expression profile and anti-proliferation effect of AURKA inhibitor in RCC cells. Immunoblot detection revealed that two RCC cell lines (ACHN and CAKI) expressed the wild type VHL gene, while the other three (A498, 769-P and 786-O) were VHL-deficient cells (Fig. 1A), consistent with reports by the National Cancer Institute using cDNA micro-arrays (genome-www.edu/nci60/).

Inhibition of cellular proliferation was evaluated using a CCK-8 kit (Beyotime Institute of Biotechnology, Haimen, China). In the present study, the AURKA-specific chemical inhibitor alisertib more readily inhibited the growth of ACHN and CAKI cells when compared with its effect in the other three RCC cell lines (Fig. 1B). IC50 values for alisertib in each cell line were as follows: ACHN, $\sim 8 \mu \mathrm{mol} / 1$; CAKI, $\sim 7.5 \mu \mathrm{mol} / 1$; $786-\mathrm{O}, \sim 0.20 \mu \mathrm{mol} / 1$; 769-P, $\sim 0.25 \mu \mathrm{mol} / 1$; A-498, $\sim 0.33 \mu \mathrm{mol} / \mathrm{l}$. We found a clear positive relationship between VHL gene expression and alisertib sensitivity (IC50) in the RCC cells. In other words, RCC cells expressing wild type VHL were more sensitive to alisertib (Fig. 1A and B). This Pearson's correlation was further confirmed in a xenograft animal model. As shown in Fig. 1C, alisertib displayed differential anti-tumor activity in CAKI and 769-P xenografts, with inhibitory rates of 36.0 and $60.7 \%$, respectively.

Alteration in alisertib anti-proliferation activity by VHL. To confirm the positive relationship between the VHL gene expression profile and alisertib sensitivity in RCC cell lines, 769-P cells were stably transfected with pCDH-VHL plasmid; cells transfected with empty pCDH-vector served as the control. As shown in Fig. 2A, VHL protein could be detected in 769-P cells transfected with pCDH-VHL. Furthermore, 769-P cells transfected with $\mathrm{pCDH}-\mathrm{VHL}$ were shown to be more resistant to alisertib than those transfected with empty vector (Fig. 2B), with the IC50 increasing from 0.25 to $1.25 \mu \mathrm{mol} / 1$.

To further explore whether there is a causal relationship between VHL expression and alisertib sensitivity, CAKI cells were transfected with shRNA directed against VHL; cells transfected with empty vector as the control. Cells were harvested $72 \mathrm{~h}$ after transfection and extracts were prepared and analyzed by western blotting. Expression of the VHL gene decreased significantly after shRNA transfection (Fig. 3A). At $24 \mathrm{~h}$ after transfection with VHL shRNA or control vector, CAKI cells were treated with alisertib for $72 \mathrm{~h}$. The cytotoxicity of alisertib was significantly higher for cells in the presence of VHL shRNA than for the control group (Fig. 3B), which indicated that decreasing the expression of VHL altered the anti-proliferation activity of alisertib.

This association was further confirmed in the animal model. As shown in Fig. 3C, alisertib displayed differential anti-tumor activity in the xenograft model of CAKI cells transfected with VHL-shRNA or control vector, with inhibitory rates of $\sim 54$ and $\sim 32 \%$, respectively (Fig. 3C). 

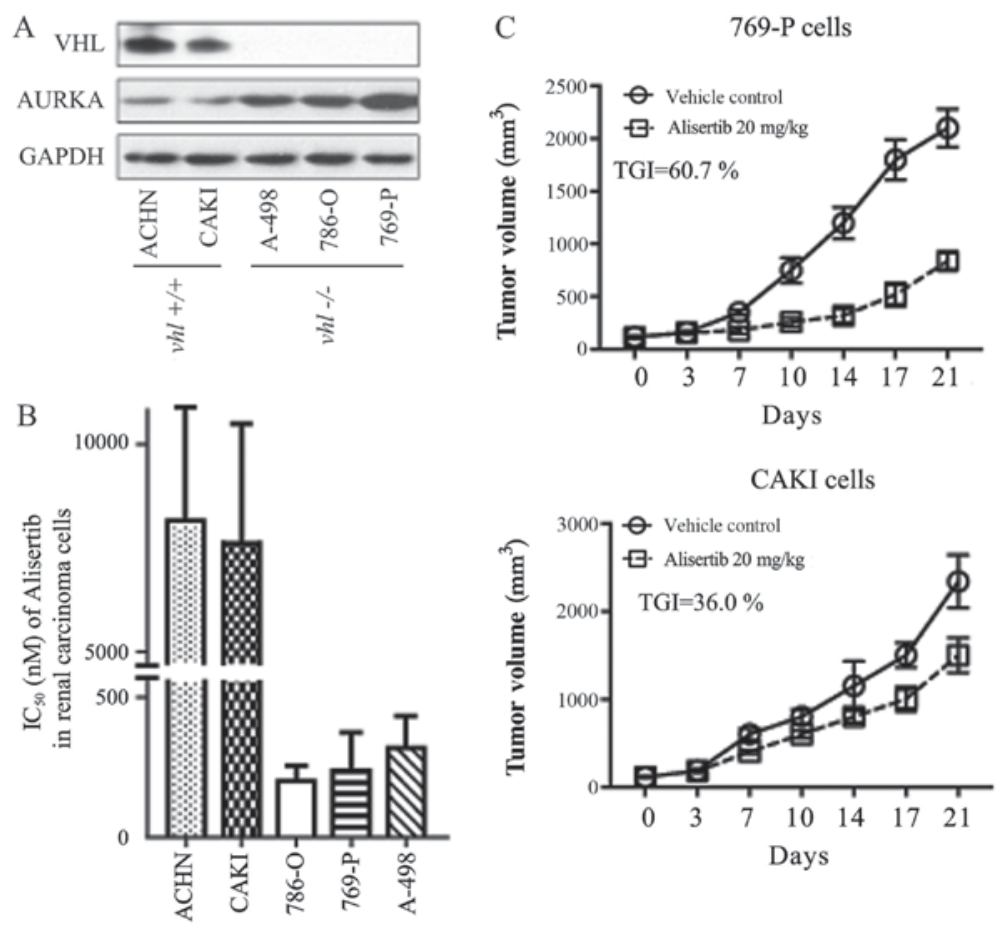

Figure 1. VHL expression profiles and alisertib anti-proliferative activities in RCC cells. (A) VHL and AURKA protein expression was detected by immunoblotting. GAPDH was employed as a loading control. (B) Anti-proliferative activity was assessed by Cell Counting Kit-8 assay following exposure to alisertib for 72 h. (C) Alisertib anti-tumor activity in vivo. VHL, von Hippel-Lindau tumor suppressor; RCC, renal cell carcinoma; AURKA, Aurora kinase A; IC50, half-maximal inhibitory concentration; TGI, tumor growth inhibition rate.
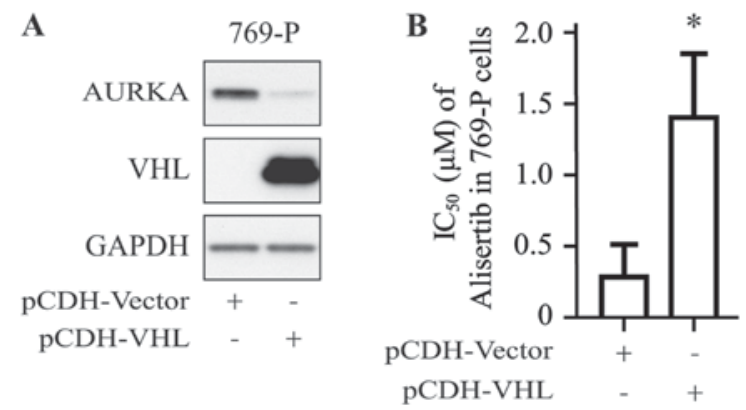

Figure 2. 769-P cells with VHL re-expression show resistance to alisertib. (A) VHL and AURKA protein expression in 769-P cells transfected with control or VHL plasmid was analyzed by immunoblotting. GAPDH was employed as a loading control. (B) The anti-proliferative activity of alisertib against cells was assessed by Cell Counting Kit -8 assay. ${ }^{~} \mathrm{P}<0.01$ vs. vector. VHL, von Hippel-Lindau tumor suppressor; AURKA, Aurora kinase A; IC50, half-maximal inhibitory concentration.

VHL down-regulates AURKA in RCC cells. According to our results, we may conclude that VHL loss makes human RCC cells more sensitive to AURKA inhibitor. This increased sensitivity might be caused by VHL down-regulating AURKA via the HIF pathway. Consistent with previous reports $(16,17)$, we showed that VHL down-regulated AURKA expression (Figs. 1A, 2A and 3A).

As reported by $\mathrm{Xu}$ et al (16), VHL inactivation induced AURKA expression in clear cell RCC (CCRCC) cells via the HIF pathway. In the present study, we also observed that hypoxic conditions could induce AURKA protein expression in RCC (Fig. 4A).

We have shown in a previous study that $\mathrm{pVHL}$ regulate AURKA directly via an HIF-independent pathway (17). As shown in Fig. 4B and C, in the present study we confirmed that VHL can promote AURKA degradation. Moreover, the proteasome inhibitor, MG-132, blocked pVHL-induced AURKA degradation. Interestingly, the AURKA protein had a shorter half-life in 769-P cells re-expressed with VHL compared with that in control cells.

\section{Discussion}

In this study, we showed that 786-O, 769-P and A498 cells deficient in the VHL gene were sensitive to alisertib, an AURKA-specific chemical inhibitor. Conversely, alisertib-resistant CAKI and ACHN cells expressed the wild type VHL gene. Re-expression or knockdown of VHL reversed the anti-proliferation activity of alisertib in RCC cells. The inverse association between VHL gene expression profile and alisertib sensitivity was confirmed in human cancer xenografts models. Taken together, we suggest that VHL loss could potentially serve as a biomarker for predicting the efficacy of AURKA inhibitors.

It is well known that VHL protein serves as a substrate recognition component of an E3-ubiquitin ligase complex that targets hypoxia inducible factor (HIF) for ubiquitination and degradation (18). In addition to HIF- $\alpha$, pVHL interacts with certain other proteins and has multiple functions, including in microtubule dynamics, cell proliferation and growth, neuronal apoptosis, extracellular matrix deposition, DNA damage response/repair and cilia maintenance. It has been extensively reported in human cancers that loss of VHL is a predictive biomarker for the efficacy of neoadjuvant therapy $(19,20)$. In the present study, we proposed the potential of VHL to be a predictive marker of response to AURKA inhibitors and tested 
$\mathbf{A}$

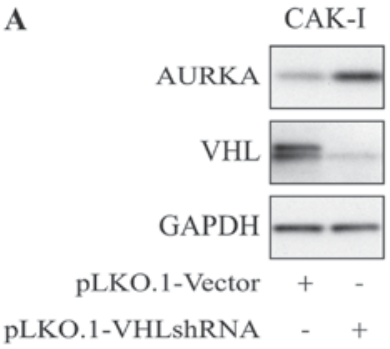

B

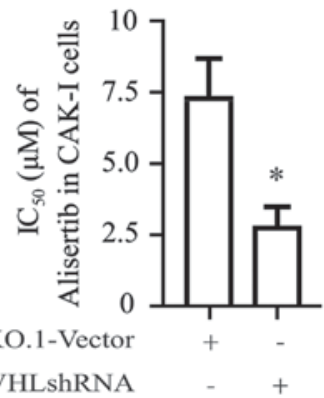

pLKO.1-VHLshRNA

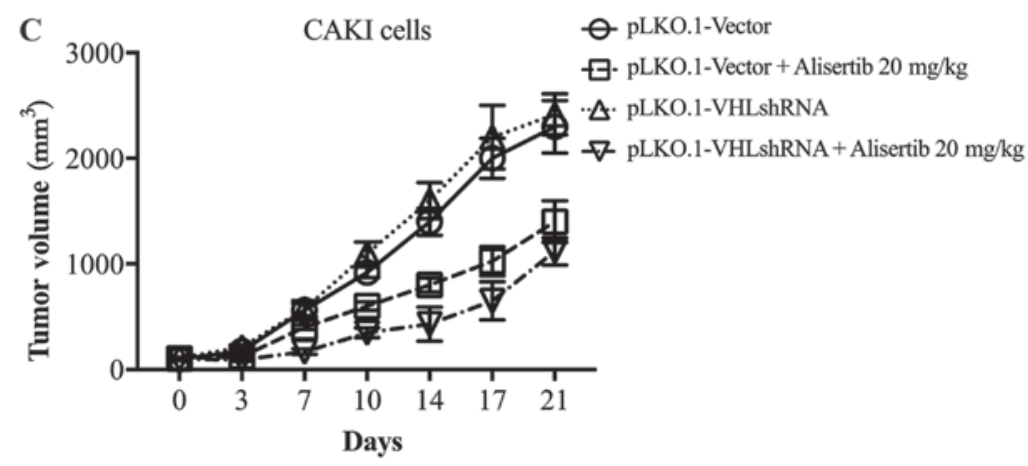

Figure 3. shRNA knockdown of VHL in CAKI cells confers decreased alisertib sensitivity. (A) VHL and AURKA protein expression in CAKI cells transfected with control or VHL shRNA was analyzed by immunoblotting. GAPDH was employed as a loading control. (B) The anti-proliferative activity of alisertib against cells was assessed by Cell Counting Kit -8 assay. ${ }^{*} \mathrm{P}<0.01$ vs. vector. (C) Inhibition of tumor growth by alisertib in xenografts of CAKI cells transfected with control or VHL shRNA. VHL, von Hippel-Lindau tumor suppressor; AURKA, Aurora kinase A; IC50, half-maximal inhibitory concentration; shRNA, short hairpin RNA.
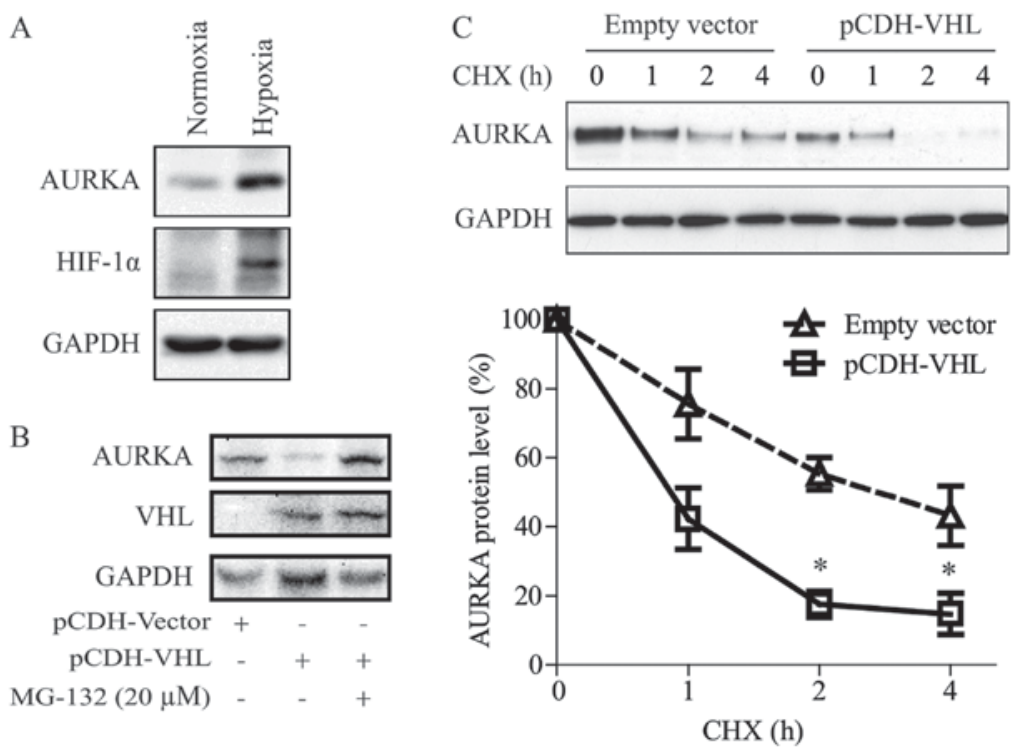

Figure 4. VHL regulates AURKA levels via HIF-dependent and -independent pathways. (A) Hypoxia could induce AURKA protein upregulation upon analysis of HIF-1 and AURKA protein expression in CAK-I cells by immunoblotting; GAPDH was employed as a loading control. (B) The 26S proteasome inhibitor MG132 $(10 \mu \mathrm{M})$ rescued the downregulation of AURKA in 769-P cells re-expressed with pVHL. "P<0.01 vs. empty vector. (C) pVHL promoted AURKA degradation. 769-p cells were transfected with either pVHL or vector plasmid. Cells were treated with $100 \mu \mathrm{g} / \mathrm{ml} \mathrm{CHX}$ and harvested at the indicated time points, and cell lysates were prepared. Proteins from cell lysates were subjected to western blotting with anti-AURKA and anti-GAPDH antibodies. Relative protein levels were plotted from the integrated optical density of the AURKA bands on the western blot (lower panel). VHL, von Hippel-Lindau tumor suppressor; AURKA, Aurora kinase A; IC50, half-maximal inhibitory concentration; HIF, hypoxia inducing factor; pVHL, VHL protein; CHX, cycloheximide.

our hypothesis in vitro and in vivo. We further speculated that pVHL regulated AURKA levels via HIF-dependent and HIF-independent pathways. As it is technically feasible and reproducible to measure VHL loss in patients, which should be easier than detecting AURKA expression levels, VHL meets the criteria to serve as a predictive marker of efficacy of alisertib-based therapy.

Our data supported that VHL loss could be potentially applied as a biomarker to predict the efficacy of AURKA inhibitors. Additional pre-clinical and clinical trials are needed 
to validate our findings. It is necessary to confirm our primary data with results obtained from the patients' samples (e.g. PDTX model) and correlate the results with the clinic pathological data.

\section{Acknowledgements}

The authors would like to thank Mr. Jian-Xing Zhang (Laboratory for Biological Medicine, School of Medicine, Taizhou University, Zhejiang, China) for their technical assistance during the animal work.

\section{Funding}

This work was supported by the Zhejiang Provincial Natural Science Foundation (grant nos. LY15H310002, LY15H310001 and LY16H310006), the National Natural Science Foundation of China (grant no. 81201530), the Public Technology Research Projects of the Science Technology Department of Zhejiang Province (grant nos. 2016C37111 and 2015C37093, and the Science Technology Department of Taizhou City (grant no. 15yw08).

\section{Availability of data and materials}

The datasets used and/or analyzed during the current study are available from the corresponding author on reasonable request.

\section{Authors' contributions}

XFD, GC and YLW designed the research. XFD, JZ and GC performed the research. XFD, GC and YLW analyzed the data, and GC and YLW wrote the manuscript.

\section{Ethics approval and consent to participate}

Animal experiments were performed according to the institutional ethical guidelines of animal care and were approved by Taizhou University (no. TZYXY2016-302).

\section{Consent for publication}

Not applicable.

\section{Competing interests}

The authors declare that they have no competing interests.

\section{References}

1. Kidney cancer update the year in review in kidney cancer. Clin Adv Hematol Oncol 13: 327-329, 2015.
2. Massari F, Di Nunno V, Ciccarese C, Graham J,Porta C, Comito F, Cubelli M, Iacovelli R and Heng DYC: Adjuvant therapy in renal cell carcinoma. Cancer Treat Rev 60: 152-157, 2017.

3. Posadas EM, Limvorasak S and Figlin RA: Targeted therapies for renal cell carcinoma. Nat Rev Nephrol 13: 496-511, 2017.

4. Cha TL, Chuang MJ, Wu ST, Sun GH, Chang SY, Yu DS, Huang SM, Huan SK, Cheng TC, Chen TT, et al: Dual degradation of aurora A and B kinases by the histone deacetylase inhibitor LBH589 induces G2-M arrest and apoptosis of renal cancer cells. Clin Cancer Res 15: 840-850, 2009.

5. Kurahashi T, Miyake H, Hara I and Fujisawa M: Significance of Aurora-A expression in renal cell carcinoma. Urol Oncol 25: 128-133, 2007.

6. Li Y, Zhou W, Wei L, Jin J, Tang K, Li C, Teh BT and Chen X: The effect of Aurora kinases on cell proliferation, cell cycle regulation and metastasis in renal cell carcinoma. Int J Oncol 41: 2139-2149, 2012.

7. Karthigeyan D, Prasad SB, Shandilya J, Agrawal S and Kundu TK: Biology of Aurora A kinase: Implications in cancer manifestation and therapy. Med Res Rev 31: 757-793, 2011.

8. Borisa AC and Bhatt HG: A comprehensive review on Aurora kinase: Small molecule inhibitors and clinical trial studies. Eur J Med Chem 140: 1-19, 2017.

9. Lim SK and Gopalan G: Aurora-A kinase interacting protein 1 (AURKAIP1) promotes Aurora-A degradation through an alternative ubiquitin-independent pathway. Biochem J 403: 119-127, 2007.

10. EckerdtF,Pascreau G,Phistry M,Lewellyn AL,DePaoli-Roach AA and Maller JL: Phosphorylation of TPX2 by Plx1 enhances activation of Aurora A. Cell Cycle 8: 2413-2419, 2009.

11. Huang Y, Li T, Ems-McClung SC, Walczak CE, Prigent C, Zhu X, Zhang $\mathrm{X}$ and Zheng Y: Aurora A activation in mitosis promoted by BuGZ. J Cell Biol 217: 107-116, 2018.

12. Korobeynikov V, Deneka AY and Golemis EA: Mechanisms for nonmitotic activation of Aurora-A at cilia. Biochem Soc Trans 45: 37-49, 2017.

13. Compérat $\mathrm{E}$, Bièche I, Dargère $\mathrm{D}$, Laurendeau $\mathrm{I}$, Vieillefond $\mathrm{A}$, Benoit G, Vidaud M, Camparo P, Capron F, Verret C, et al: Gene expression study of Aurora-A reveals implication during bladder carcinogenesis and increasing values in invasive urothelial cancer. Urology 72: 873-877, 2008.

14. Goktas S, Yildirim M, Suren D, Alikanoglu AS, Dilli UD, Bulbuller N, Sezer C and Yildiz M: Prognostic role of Aurora-A expression in metastatic colorectal cancer patients. J BUON 19: 686-691, 2014

15. Twu NF, Yuan CC, Yen MS, Lai CR, Chao KC, Wang PH, Wu $\mathrm{HH}$ and Chen YJ: Expression of Aurora kinase A and B in normal and malignant cervical tissue: High Aurora A kinase expression in squamous cervical cancer. Eur J Obstet Gynecol Reprod Biol 142: 57-63, 2009.

16. Xu J, Li H, Wang B, Xu Y, Yang J, Zhang X, Harten SK, Shukla D, Maxwell PH, Pei D and Esteban MA: VHL inactivation induces HEF1 and Aurora kinase A. J Am Soc Nephrol 21: 2041-2046, 2010.

17. Hasanov E, Chen G, Chowdhury P, Weldon J, Ding Z, Jonasch E, Sen S, Walker CL and Dere R: Ubiquitination and regulation of AURKA identifies a hypoxia-independent E3 ligase activity of VHL. Oncogene 36: 3450-3463, 2017.

18. Gossage L, Eisen T and Maher ER: VHL, the story of a tumour suppressor gene. Nat Rev Cancer 15: 55-64, 2015.

19. Kim BJ, Kim JH, Kim HS and Zang DY: Prognostic and predictive value of VHL gene alteration in renal cell carcinoma: A meta-analysis and review. Oncotarget 8: 13979-13985, 2017.

20. Ferchichi I, Kourda N, Sassi S, Romdhane KB, Balatgi S, Cremet JY, Prigent C and Elgaaied AB: Aurora A overexpression and pVHL reduced expression are correlated with a bad kidney cancer prognosis. Dis Markers 33: 333-340, 2012. 